EPJ Web of Conferences 116, 06004 (2016)

DOI: $10.1051 /$ epjconf/201611606004

(C) Owned by the authors, published by EDP Sciences, 2016

\title{
Moon shadow observation with ANTARES and KM3NeT neutrino telescope
}

\author{
Matteo Sanguineti ${ }^{1,2, a}$ and Carla Distefano ${ }^{3, \mathrm{~b}}$ \\ ${ }^{1}$ INFN - Sezione di Genova, via Dodecaneso 33, 16146 Genova, Italy \\ 2 Dipartimento di Fisica dell'Università, via Dodecaneso 33, 16146 Genova, Italy \\ ${ }^{3}$ INFN - Laboratori Nazionali del Sud (LNS), via S. Sofia 62, 95123 Catania, Italy
}

\begin{abstract}
The ANTARES detector is the largest neutrino telescope currently in operation in the Northern Hemisphere. The search for point-like neutrino sources is one of the main goals of ANTARES, so a reliable way to evaluate the detector pointing performance is needed. The Moon shadow measurement, i.e. the deficit in the atmospheric muon flux in the direction of the Moon induced by absorption of cosmic rays, allows the estimation of the angular resolution and the pointing accuracy. The 2007-2012 ANTARES data set shows a significance of Moon shadowing around $3 \sigma$. This is the first measurement of the ANTARES angular resolution and absolute pointing for atmospheric muons using a celestial calibration source. The same approach has been used to estimate also the expected Moon shadow significance with the KM3NeT-ARCA detector, the future cubic kilometre scale detector that will be installed in Sicily.
\end{abstract}

\section{Introduction}

Neutrino telescopes have opened a new era of observation of the Universe. Neutrinos are chargeless, weakly interacting particles that can cross dense matter or radiation fields without being absorbed for cosmological distances. Therefore they can provide unperturbed information on the far Universe and the interior of astrophysical sources.

The ANTARES neutrino telescope [1] is designed for the identification of point-like sources of high energy cosmic neutrinos, like starburst galaxies, GRBs, Supernova remnants and AGNs. Following the experience from the ANTARES experiment, the KM3NeT collaboration has started the construction of the next generation deep-sea neutrino telescope. It will be a cubic kilometre scale detector with several hundreds of detection units.

The pointing accuracy and the angular resolution of these detectors are critically important for the detection of point-like sources and a proper way to evaluate these performances is needed.

A deficit in the event density of the atmospheric muon flux corresponding to the direction of the Moon disk is expected due to the absorption of cosmic rays by the satellite. Several experiments, like

\footnotetext{
a e-mail: matteo.sanguineti@ge.infn.it

b e-mail: distefano_c@lns.infn.it
}

This is an Open Access article distributed under the terms of the Creative Commons Attribution License 4.0, which permits unrestricted use, distribution, and reproduction in any medium, provided the original work is properly cited. 


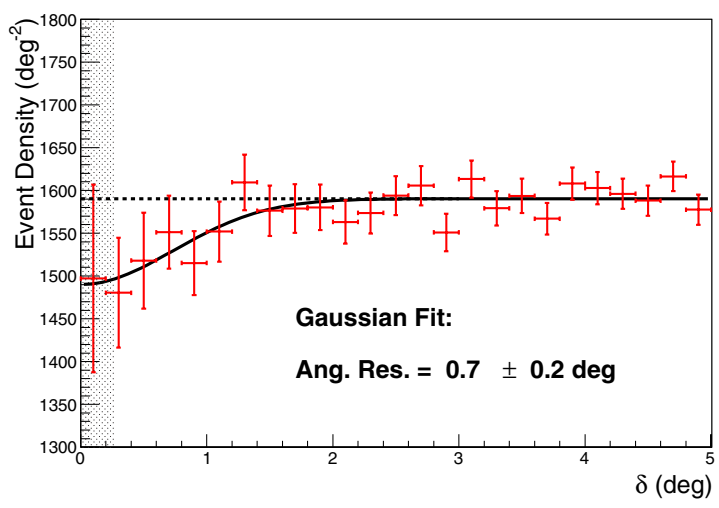

Figure 1. Event density of muons after selection cut versus the angular distance from the Moon centre.

CYGNUS [2], TIBET [3], CASA [4], MACRO [5], SOUDAN [6] , ARGO [7] and IceCube [8], used the so-called Moon shadow effect to test the pointing performance of the detector.

In this work we exploit this technique to measure the ANTARES angular resolution for atmospheric down-going muons and we show the expected performances of the KM3NeT-ARCA detector.

\section{Detection of the Moon shadow with ANTARES}

The MUPAGE [9] code has been used to simulate the atmospheric muon events, where geo-magnetic deflection is not taken into account. The correction of the muon trajectory is negligible at detector level because only low energy muons (absorbed before reaching the detector) are strongly deflected [10], so the geo-magnetic effect can be neglected in this analysis. The detector response to the Cherenkov light emitted by the muon tracks is simulated [11]. Finally a robust track fitting procedure based on a maximum likelihood method is used to reconstruct the direction of the atmospheric muon tracks [12]. The shadowing effect is simulated rejecting the muons generated within the Moon disk $\left(R_{\text {Moon }}=0.259^{\circ}\right)$. The simulation live time is 2080 days in this data analysis (years 2007-2012).

The region of the sky around the Moon centre is divided in concentric rings with increasing radius (ring size $=0.2^{\circ}$ ). Event tracks detected when the Moon is above the Horizon and reconstructed as down-going are selected.

A test statistic function $t$ is defined as:

$$
t=\sum_{\text {rings }} \frac{\left(n_{m}-n_{\text {exp,NO_Moon }}\right)^{2}}{n_{\text {exp,NO_Moon }}}-\frac{\left(n_{m}-n_{\text {exp }, \text { Moon }}\right)^{2}}{n_{\text {exp,Moon }}},
$$

where the sum is over all the rings around the Moon centre; $n_{m}$ is the number of events detected in a ring, $n_{\text {exp,Moon }}$ is the expected number of events in "Moon shadow" hypothesis and $n_{\text {exp,NO}}$ Moon is the expected number of events in "no Moon shadow" hypothesis [13].

The significance of the Moon shadow deficit was estimated optimising the event selection using the test statistic $t$ (Eq. (1)). The expected Moon shadow significance with optimized event selection is $2.9 \sigma$. The optimized selection criteria were applied to the data showing a 3.1 $\sigma$ significance for the rejection of "No Moon shadow" hypothesis. The event density for selected muons as a function of the angular distance from the Moon centre is shown in Fig. 1. 


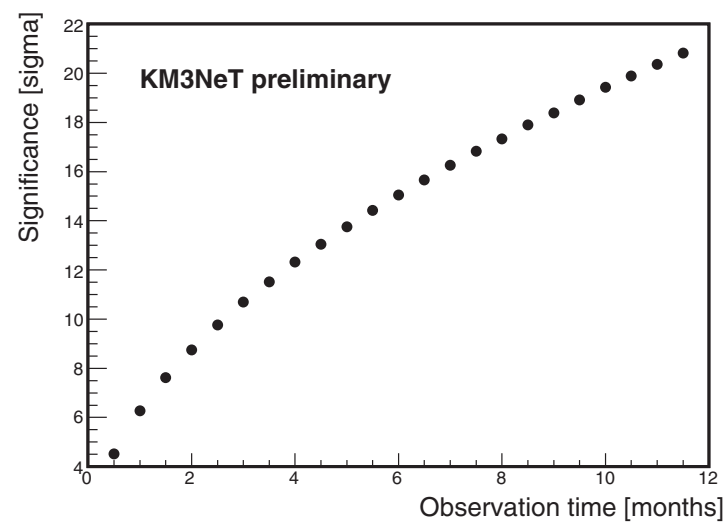

Figure 2. Moon shadow significance as a function of the measure time based on a simulation of the KM3NeTARCA detector with one building block.

The detector angular resolution can be evaluated fitting the event density with the formula:

$$
\frac{\mathrm{d} n}{\mathrm{~d} \delta^{2}}=k\left(1-\frac{R_{M o o n}^{2}}{2 \varsigma^{2}} e^{-\frac{\delta^{2}}{2 \varsigma^{2}}}\right)
$$

where $R_{\text {Moon }}=0.259^{\circ}$ is the Moon angular radius and $\delta$ is the angular distance from the Moon centre. The fit free parameters $k$ and $\varsigma$ are respectively the off-source density level and the detector angular resolution. We have assumed a Gaussian shape for the detector point spread function [6]. From the fit we can estimate the angular resolution: $\varsigma=0.7^{\circ} \pm 0.2^{\circ}$.

\section{Simulation of the Moon shadow with KM3NeT-ARCA}

The same approach of Sect. 2 has been used to estimate the expected Moon shadow significance with the KM3NeT-ARCA detector.

A single building block of KM3NeT-ARCA has been considered. It is composed of 115 detection units and each unit has 18 digital optical modules (DOMs). The spacing between the DOMs is $36 \mathrm{~m}$, whereas the spacing between the detection units is $90 \mathrm{~m}$.

In Fig. 2 the Moon shadow significance is shown as a function of the measure time.

The expected significance reached in one year is around $21 \sigma$. The corresponding plot of event density for selected muons as a function of the angular distance from the Moon centre is shown in Fig. 3. The fitted angular resolution (Eq. (2)) is $\varsigma=0.43^{\circ} \pm 0.01^{\circ}$.

\section{Conclusions}

The Moon shadow observation is a good way to estimate the pointing performance of neutrino telescopes. It has been observed with a 3.1 $\sigma$ significance in the ANTARES data sample 2007-2012. The Moon shadow profile has been fitted assuming a Gaussian shape for the detector point spread function, hence we derived the angular resolution for the atmospheric muon flux: $0.7^{\circ} \pm 0.2^{\circ}$. The simulation of 


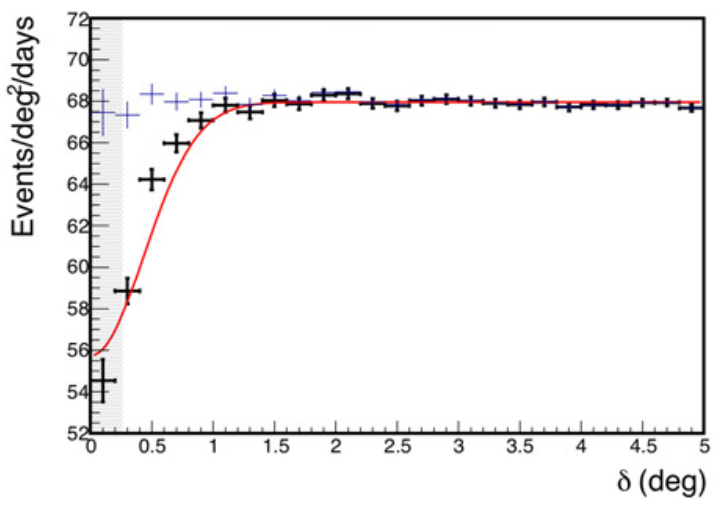

Figure 3. Expected event density of muons after selection cut versus the angular distance from the Moon centre.

the Moon shadow effect with the KM3NeT-ARCA detector predicts a $21 \sigma$ significance in one year and the expected angular resolution for down-going muons is $0.43^{\circ} \pm 0.01^{\circ}$.

\section{References}

[1] M. Ageron et al. (ANTARES), Nucl. Instrum. Meth. A656, 11 (2011), 1104.1607

[2] D.E. Alexandreas et al. (CYGNUS), Phys. Rev. D43, 1735 (1991)

[3] M. Amenomori et al. (Tibet AS gamma), Phys. Rev. D47, 2675 (1993)

[4] A. Borione et al., Phys. Rev. D49, 1171 (1994)

[5] M. Ambrosio et al. (MACRO), Phys. Rev. D59, 012003 (1999), hep-ex/9807006

[6] J.H. Cobb et al. (Soudan 2), Phys. Rev. D61, 092002 (2000), hep-ex/9905036

[7] B. Bartoli et al., Phys. Rev. D84, 022003 (2011), 1107 . 4887

[8] M.G. Aartsen et al. (IceCube), Phys. Rev. D89, 102004 (2014), 1305.6811

[9] G. Carminati, A. Margiotta, M. Spurio, Comput. Phys. Commun. 179, 915 (2008), 0802.0562

[10] C. Distefano (ANTARES), Nucl. Instrum. Meth. A626-627, S223 (2011)

[11] J. Brunner (ANTARES), Antares simulation tools, in Technical aspects of a Very Large Volume Neutrino Telescope in the Mediterranean Sea. Proceedings, Workshop, VLVnuT Workshop, Amsterdam, Netherlands, October 5-8, 2003 (2003), pp. 109-113, http://www.vlvnt.nl/proceedings/

[12] A. Heijboer, Ph.D. thesis, Amsterdam U. (2004), http://www.nikhef.nl/t61/thesis/ index.html

[13] M. Sanguineti, Moon shadow observation with the ANTARES neutrino telescope, in Proceedings, 34th International Cosmic Ray Conference (ICRC 2015) (2015), Vol. ICRC2015, http://pos.sissa.it/cgi-bin/reader/conf. cgi?confid=236 